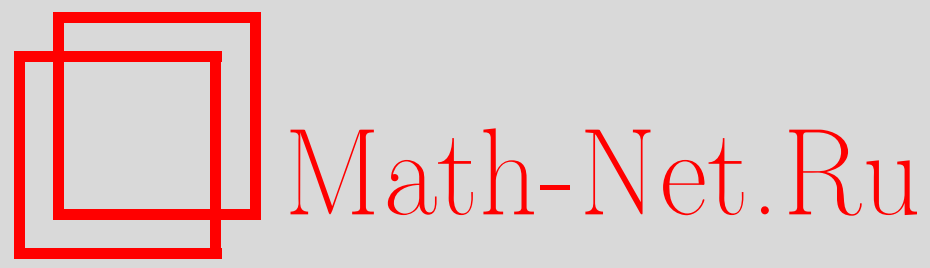

В. М. Бухштабер, Д. В. Лейкин, В. З. Энольский, Униформизация многообразий Якоби тригональных кривых и нелинейные дифференциальные уравнения, Функи. анализ и его прил., 2000, том 34, выпуск 3, 1-16

DOI: https://doi.org/10.4213/faa307

Использование Общероссийского математического портала MathNet.Ru подразумевает, что вы прочитали и согласны с пользовательским соглашением

http://www . mathnet.ru/rus/agreement

Параметры загрузки:

IP: 35.174 .16 .151

26 апреля 2023 г., 18:12:30

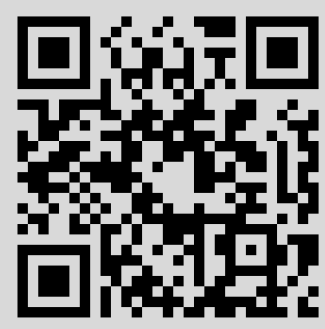


Функииональный анализ и его приложения

2000, т. 34, вып. 3, с. 1-16

УДК $512.742+517.957$

\title{
Униформизация многообразий Якоби тригональных кривых и нелинейные дифференциальные уравнения
}

\author{
(c) 2000. В. М. БУХштАБЕР, Д. В. ЛЕЙКИН, В. З. ЭНОЛЬСКИй
}

\section{Введение}

Настоящая работа посвящена развитию метода интегрирования нелинейных дифференциальных уравнений в частных производных на основе униформизации многообразий Якоби алгебраических кривых ю-функциями многих переменных, начала теории которых были заложены Клейном. Наши исследования в этом направлении (см. обзор [3]) мотивированы тем, что при такой униформизации естественно и явно возникают важнейшие уравнения математической физики.

Клейн [10] предложил строить теорию аналогов эллиптической $\sigma$-функции Вейерштрасса для кривых рода $>1$ как теорию функций нескольких переменных, родственных $\theta$-функциям, обладающих дополнительным свойством модулярной инвариантности. Дифференциальное поле абелевых функций на многообразии Якоби кривой порождается производными порядка $>1$ логарифма $\sigma$-функции, т. е. функциями

$$
\wp_{i_{1}, i_{2}, \ldots, i_{k}}(\boldsymbol{u}, \boldsymbol{\lambda})=-\frac{\partial^{k} \log \sigma(\boldsymbol{u}, \boldsymbol{\lambda})}{\partial u_{i_{1}} \ldots \partial u_{i_{k}}}, \quad k \geqslant 2 .
$$

В работе авторов [3] для гиперэллиптических кривых произвольного рода $g$ предъявлены $3 g$ базисных $\wp$-функций дифференциального поля функций на якобиане, для которых в явном виде выписаны базисные алгебраические соотношения. Для случая $g=2$ эти результаты можно извлечь из работы Бейкера [1], теория для $g>2$, построенная авторами, потребовала существенного развития классических методов. Необходимо отметить, что в работах классического периода отсутствует всякое сопоставление полученных дифференциальных соотношений с задачами теории дифференциальных уравнений в частных производных, несмотря на то что полученные для случая $g=2$ в [1] соотношения приводят к решениям дифференциальных уравнений (например, таких, как sine-Gordon), актуальных уже в то время.

Наш подход к теории в общем случае опирается на модели кривых, которые в работе [4] названы $(n, s)$-кривыми. Гиперэллиптические кривые рода $g$ в этой терминологии являются $(2,2 g+1)$-кривыми. Ниже мы излагаем результаты для $(3, g+1)$-кривых, которые являются так называемыми тригональныли кривыми рода $g$. В классе $(n, s)$-кривых случаи $n=2$ и $n=3$ выделены тем, что только для них выполняются неравенства $n \leqslant g=(n-1)(s-1) / 2<s$ для рода кривой $g$, играющие важную роль при построении теории.

В $\S 1$ содержатся необходимые сведения и вводится важное для дальнейшего понятие универсального пространства $g$-х симметрических степеней тригональных кривых $\mathrm{U}_{g}$. Основным результатом $\S 2$ является теорема 2.6 , дающая 
явное описание пространства $\mathrm{U}_{g}$ как алгебраического подмногообразия в комплексном линейном пространстве размерности $4 g$ или $4 g+2$, если $g=3 \ell$ или $g=3 \ell+1$. В $\S 3$ получена явная реализация многообразия Куммера тригональной кривой как алгебраического подмногообразия в $\mathbb{C}^{g(g+1) / 2}$, заданого системой $(g-1) g / 2$ полиномиальных уравнений степени не выше 5 . Эффективность нашего подхода демонстрируется на примере негиперэллиптической кривой рода $3 . \mathrm{B} \oint 4$ выделен набор из $4 g$ ю-функций на многообразии Якоби тригональной кривой, которые униформизуют построенные в $\S 2$ алгебраические многообразия. В этом же параграфе содержатся все необходимые сведения о $\sigma$-функции. В $\S 5$ мы анализируем полученные дифференциальные соотношения с точки зрения теории дифференциальных уравнений в частных производных. В частности, сравнивая с аналогичными результатами для гиперэллиптического случая, мы показываем, что теория ю-функций является эффективным инструментом характеризации якобианов аналитическими средствами.

Авторы выражают благодарность С. П. Новикову, оказавшему большое влияние на формирование нашей точки зрения на предмет данного исследования.

\section{$\S 1$. Универсальное пространство $g$-х симметрических степеней тригональных кривых}

Пусть $\operatorname{gcd}(g+1,3)=1$. Для $g$ возможны значения $g=3 \ell$ и $g=3 \ell+1$, где $\ell=1,2, \ldots$.

ОпРеДЕлЕниЕ 1.1. Будем называть тригональныл линейный по параметрам $\boldsymbol{\lambda}=\left\{\lambda_{0}, \lambda_{3}, \ldots\right\}$ полином $f_{g}(x, y ; \boldsymbol{\lambda})$ с коэффициентами при старших степенях $x$ и $y$, равными -1 и 1 соответственно, который в градуировке $\operatorname{deg} x=3$, $\operatorname{deg} y=g+1$ и $\operatorname{deg} \lambda_{i}=3 g+3-i>0$ однороден веса $\operatorname{deg} f_{g}=3(g+1)$.

Из определения следует, что тригональный полином имеет вид

$$
\begin{aligned}
f_{g}(x, y ; \boldsymbol{\lambda})=y^{3}-y^{2}( & \left.\sum_{j=0}^{[(g+1) / 3]} \lambda_{2 g+2+3 j} x^{j}\right) \\
& -y\left(\sum_{j=0}^{[(2 g+2) / 3]} \lambda_{g+1+3 j} x^{j}\right)-\left(x^{g+1}+\sum_{j=0}^{g} \lambda_{3 j} x^{j}\right)
\end{aligned}
$$

и зависит от набора из $2 g+3$ параметров $\boldsymbol{\lambda}=\left\{\lambda_{0}, \lambda_{3}, \ldots, \lambda_{3 g+1}, \lambda_{3 g+2}\right\}$. Множество индексов у элементов набора $\boldsymbol{\lambda}$ пробегает все числа вида $3 a+(g+1) b$ (при целых неотрицательных $a$ и $b$ ) в интервале от 0 до $3 g+2$; при этом имеется в точности $g$ пропусков, так называемых пробелов, которые образуют последовательность Вейерштрасса (более детально см. [4]).

ОПРЕДЕЛЕниЕ 1.2. Будем называть пространством модулей тригональных полиномов веса $3(g+1)$ градуированное пространство $\mathrm{M}_{g}$, точками которого являются указанные выше наборы $\boldsymbol{\lambda}$, т. е. $\mathrm{M}_{g} \cong \mathbb{C}^{2 g+3}$.

При числовых значениях параметров $\left\{\lambda_{k}\right\}$ множество $\left\{(x, y) \in \mathbb{C}^{2} \mid f_{g}(x, y ; \boldsymbol{\lambda})\right.$ $=0\}$ нулей тригонального полинома представляет собой аффинную модель плоской тригональной кривой $V(x, y)$ рода не выше $g$. При условии невырожденности полинома $f_{g}$, т. е. когда значения параметров $\left\{\lambda_{k}\right\}$ таковы, что трой- 
ка полиномов $\left\{f_{g}(x, y ; \boldsymbol{\lambda}), \frac{\partial}{\partial y} f_{g}(x, y ; \boldsymbol{\lambda}), \frac{\partial}{\partial x} f_{g}(x, y ; \boldsymbol{\lambda})\right\}$ не имеет общих нулей, кривая $V(x, y)$ неособа, и ее род равен $g$.

Любая иелая рациональная функиия на кривой $V(x, y)$, т. е. функция, принимающая конечные значения в точках аффинной модели кривой $V$, может быть записана в так называемом нормальном виде $p(x, y)=p_{2}(x) y^{2}+p_{1}(x) y+p_{0}(x)$, где $p_{0}(x), p_{1}(x)$ и $p_{1}(x)$ - некоторые полиномы от $x$. Наибольший из весов мономов $x^{i} y^{j}$, встречающихся в полиноме $p(x, y)$, называется порядком этой целой рациональной функции. В дальнейшем нам понадобятся следующие факты (см., например, $[2,12]):$ число нулей целой рациональной функции $p(x, y)$ на кривой $V(x, y)$, т. е. число пар $\left\{(\xi, \eta) \in \mathbb{C}^{2} \mid f_{g}(\xi, \eta ; \boldsymbol{\lambda})=0, p(\xi, \eta)=0\right\}$, равно ее порядку; целая рациональная функция на кривой, имеющая $2 g+n$ нулей $(n \geqslant 0)$, определяется набором из $g+n$ параметров.

Как известно $[6,11]$, отображение Абеля переводит $k$-ю симметрическую степень кривой $V(x, y)$ в ее многообразие Якоби $\operatorname{Jac}(V)$. При $k$, равном (или большем) роду кривой, отображение Абеля является отображением на. Ниже нам понадобится следующий вариант теоремь Абеля: на множестве нулей целой рациональной функции на кривой отображение Абеля принимает значение нуль.

Далее символом $(X)^{k}$ будем обозначать $k$-ю симметрическую степень пространства $X$.

ОПРЕДЕЛЕНИЕ 1.3. Универсальным пространством $g$-х симметрических степеней тригональных кривых $V$ называется пространство $\mathrm{U}_{g} \subset\left(\mathbb{C}^{2}\right)^{g} \times \mathrm{M}_{g}$ всех наборов $\left(\left\{\left(x_{i}, y_{i}\right)\right\}_{i=1, \ldots, g} ; \boldsymbol{\lambda}\right)$, таких, что $f_{g}\left(x_{i}, y_{i} ; \boldsymbol{\lambda}\right)=0$ для всех $i=$ $1, \ldots, g$.

Универсальным расслоением $g$-х симметрических степеней тригональньх кривых $V$ называется тройка $\left(\mathrm{U}_{g}, \mathrm{M}_{g}, p_{\mathrm{U}}\right)$, где $p_{\mathrm{U}}$ - каноническая проекция.

\section{§. Матричная конструкция}

Обозначим через $\boldsymbol{W}_{g}(x, y)$ вектор $\left(w_{1}, w_{2}, \ldots, w_{g+3}\right)^{T}$, образованный однородными элементами $w_{k}=x^{\alpha} y^{\beta}$, упорядоченными по возрастанию веса $\operatorname{deg} w_{k}$ $=3 \alpha+\beta(g+1)$ при условии, что $0 \leqslant \operatorname{deg} w_{k} \leqslant 2 g+2$. Пусть $H=\left\{h_{i, j}\right\}$, $i, j=1, \ldots, g+3$, - симметрическая матрица, матричные элементы которой являются однородными элементами, $\operatorname{deg} h_{i, j}=4 g+2-\operatorname{deg} w_{i}-\operatorname{deg} w_{j}$, подходящего градуированного кольца, и, таким образом, для $H$ выполняется соотношение $\operatorname{deg}\left(\boldsymbol{W}_{g}^{T} H \boldsymbol{W}_{g}\right)=4 g+2$. Обозначим пространство симметрических матриц, градуированных таким образом, через $\mathscr{H}$.

Для любой матрицы $M$ положим $r(x, y ; M)=\frac{\partial}{\partial y}(0, \ldots, 0,1) M \boldsymbol{W}_{g}(x, y)$. Заметим, что если $H \in \mathscr{H}$, то $\operatorname{deg} r(x, y ; H)=g-1$.

ОПрЕДЕЛЕНИЕ 2.1. Обозначим через $\mathscr{T}_{g} \subset \mathscr{H} \times \mathrm{M}_{g}$ подпространство пар $H \in \mathscr{H}$ и $\lambda \in \mathrm{M}_{g}$, таких, что имеет место соотношение

$$
\boldsymbol{W}_{g}^{T} H \boldsymbol{W}_{g}=2 r(x, y ; H) f_{g}(x, y ; \boldsymbol{\lambda}) .
$$

Таким образом, определено расслоение $\left(\mathscr{T}_{g}, \mathrm{M}_{g}, p_{\mathscr{T}}\right)$, где $p_{\mathscr{T}}$ - каноническая проекция. 
Учитывая, что тригональный полином включает параметры $\left\{\lambda_{k}\right\}$ только положительных весов, из определения получаем, что матрицы из $\mathscr{T}_{g}$ имеют вид

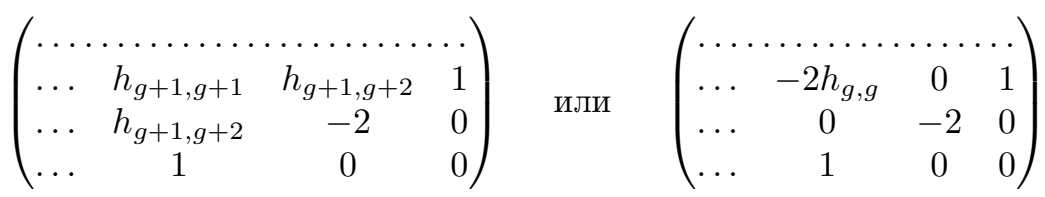

при $g=3 \ell+1$ или $g=3 \ell$ соответственно. Обозначим через $p_{1}$ и $p_{2}$ проекции произведения $\mathscr{H} \times \mathrm{M}_{g}$ на первый и второй сомножители.

Лемма 2.1. Композииия вложения $\mathscr{T}_{g} \subset \mathscr{H} \times \mathrm{M}_{g}$ с проекиией $p_{1}$ является вложением, а его композиция с проекиией $p_{2}$ совпадает $c p_{\mathscr{T}}$ и является отображением на.

ДокАЗАтЕЛЬСтво. 1. Нам нужно показать, что если для данной матрицы $H$ существует тригональный полином, удовлетворяющий соотношению (2.1), то этот полином определен однозначно. Действительно, в противном случае мы пришли бы к соотношению $r(x, y ; H)\left(f\left(x, y ; \boldsymbol{\lambda}_{1}\right)-f\left(x, y ; \boldsymbol{\lambda}_{2}\right)\right)=0$ для любых $(x, y) \in \mathbb{C}^{2}$, которое не может выполняться, если $\boldsymbol{\lambda}_{1} \neq \boldsymbol{\lambda}_{2}$.

2. Нужно показать, что при заданном $\lambda \in \mathrm{M}_{g}$ найдется хотя бы одно решение $H$ уравнения (2.1). Пусть $q$ - некоторый однородный полином веса $g-1$ от $x$ и $y$ с коэффициентами неотрицательного веса. Поскольку $\operatorname{deg} y=$ $g+1>g-1$, такой полином не содержит $y$. Пусть $M(q, \boldsymbol{\lambda})$ - решение уравнения $\boldsymbol{W}_{g}^{T} M \boldsymbol{W}_{g}-2 q(x) f_{g}(x, y ; \boldsymbol{\lambda})=0$ в классе симметрических матриц с матричными элементами неотрицательного веса. Множество решений этого уравнения, очевидно, непусто. Рассмотрев коэффициент при старшей степени $y$, получаем, что $r(x, y ; M(q, \boldsymbol{\lambda}))=q(x)$.

Введем подпространство матриц $\mathscr{H}_{4}=\{H \in \mathscr{H} \mid \operatorname{rank} H \leqslant 4\}$.

Лемма 2.2. Пусть симметрическая $(N+k) \times(N+k)$-матрица $M=\left\{m_{i, j}\right\}$ имеет невьрожденную $k \times k$-подматричу $\Delta$, которую для определенности мьи будем считать находящейся в нижнем правом углу. Тогда для того чтобь матрица $M$ имела ранг не более $k+1$, необходимо и достаточно, чтобь существовал вектор $\boldsymbol{z}=\left(z_{1}, \ldots, z_{N}\right)^{T}$, такой, что

$$
m_{i, j}=\frac{1}{\operatorname{det} \Delta} \operatorname{det}\left(\begin{array}{cc}
z_{i} z_{j} & \boldsymbol{\mu}_{i} \\
\boldsymbol{\mu}_{j}^{T} & \Delta
\end{array}\right), \quad 1 \leqslant i, j \leqslant N
$$

əде $\boldsymbol{\mu}_{r}=\left(m_{r, N+1}, m_{r, N+2}, \ldots, m_{r, N+k}\right)$.

Данная лемма является непосредственным следствием тождества Сильвестра для составных детерминантов [8]. Заметим, что вектор $\boldsymbol{z}$ в лемме 2.2 определен с точностью до знака. Обозначим через $\operatorname{Sym}^{*}(k, \mathbb{C})$ множество невырожденных симметрических $k \times k$-матриц и через $\operatorname{Sym}_{r}(k, \mathbb{C})$ множество симметрических $k \times k$-матриц ранга не более $r$, таких, что $(r-1) \times(r-1)$-подматрица, находящаяся в правом нижнем углу, невырожденна. Имеем

СледСтвиЕ 2.3. Отображение

$$
\phi: \mathbb{C}^{N} \times \mathbb{C}^{N k} \times \operatorname{Sym}^{*}(k, \mathbb{C}) \rightarrow \operatorname{Sym}_{k+1}(N+k, \mathbb{C}),
$$


заданное формулой

$$
\phi\left(\boldsymbol{z},\left(\boldsymbol{\nu}_{j}\right)_{j=1, \ldots, k}, \Delta\right)=\left(\begin{array}{cc}
\left\{m_{i, k}\right\} & \left(\boldsymbol{\nu}_{j}\right) \\
\left(\boldsymbol{\nu}_{j}\right)^{T} & \Delta
\end{array}\right),
$$

где $m_{i, k}$ въичисляются согласно формуле (2.3) при $\boldsymbol{\mu}_{r}=\left(\nu_{r, j}\right)_{j=1, \ldots, k}$, представляет собой двулистное накрьттие, разветвленное вдоль подмногообразия $\boldsymbol{z}=0$.

Положим $\Delta=\Delta_{g}$ равным соответствующему блоку из $(2.2)$ и зафиксируем вложение $\mathbb{C}^{g} \times \mathbb{C}^{3 g} \times \mathbb{C}^{\delta} \rightarrow \mathbb{C}^{g} \times \mathbb{C}^{3 g} \times \operatorname{Sym}^{*}(3, \mathbb{C})$, где $\delta=2$ при $g=3 \ell+1$ и $\delta=0$ при $g=3 \ell$. Получаем параметризацию $\varphi: \mathbb{C}^{g} \times \mathbb{C}^{3 g} \times \mathbb{C}^{\delta} \rightarrow \mathscr{H}_{4}$. Матрица $H=$ $\varphi\left(\boldsymbol{z},\left(\boldsymbol{\gamma}_{1}, \boldsymbol{\gamma}_{2}, \boldsymbol{\gamma}_{3}\right), \Delta_{g}\right)$ вычисляется по формуле $(2.4)$ при $k=3$ и $N=g$. Отметим, что отображение $\varphi$ согласуется с введенной выше градуировкой матричных элементов $h_{i, j}$, если принять следующее соглашение: $\operatorname{deg} z_{i}=2 g+1-\operatorname{deg} w_{i}$ и $\operatorname{deg} \gamma_{i, r}=2 g+3-r-\operatorname{deg} w_{i}$.

Для заданного набора $\left(\boldsymbol{z},\left(\boldsymbol{\gamma}_{1}, \gamma_{2}, \gamma_{3}\right), \Delta_{g}\right)$ определим четыре полинома $\chi(x, y)$, $\rho^{ \pm}(x, y)$ и $\tau(x, y)$ формулой

$$
\left(\chi(x, y), \rho^{ \pm}(x, y), \tau(x, y)\right)^{T}=\left(\left(\boldsymbol{\gamma}_{1}, \boldsymbol{\gamma}_{2} \pm \boldsymbol{z}, \boldsymbol{\gamma}_{3}\right)^{T}, \Delta_{g}\right) \boldsymbol{W}_{g}(x, y)
$$

По построению старшие мономы полиномов $\chi(x, y), \rho^{ \pm}(x, y)$ и $\tau(x, y)$ имеют соответственно веса $2 g+2,2 g+1$ и $2 g$. При этом переменная $y$ входит в полиномы $\tau(x, y)$ и $\rho^{ \pm}(x, y)$ линейно. Из определения отображения $\varphi$ и формулы (2.5) вытекает

ЛЕммА 2.4. Для $H=\varphi\left(\boldsymbol{z},\left(\boldsymbol{\gamma}_{1}, \boldsymbol{\gamma}_{2}, \boldsymbol{\gamma}_{3}\right), \Delta_{g}\right)$ имеет место соотношение

$$
\boldsymbol{W}_{g}^{T} H \boldsymbol{W}_{g}=-\frac{1}{2} \rho^{+} \rho^{-}+\tau\left(2 \chi+\frac{1}{2} h_{g+1, g+2}\left(\rho^{+}+\rho^{-}\right)-\left(h_{g+1, g+1}+\frac{1}{2} h_{g+1, g+2}^{2}\right) \tau\right),
$$

причем $r(x, y ; H)=\frac{\partial}{\partial y} \tau(x, y)$ и $r(x, y ; H)$ не зависит от $y$.

Пусть $\boldsymbol{Z}=\left(\boldsymbol{z},\left(\boldsymbol{\gamma}_{1}, \boldsymbol{\gamma}_{2}, \boldsymbol{\gamma}_{3}\right), \Delta_{g}\right)$ - некоторая точка из $\mathbb{C}^{g} \times \mathbb{C}^{3 g} \times \mathbb{C}^{\delta}$. Запишем правую часть равенства (2.6) в виде

$$
\boldsymbol{W}_{g}^{T} \varphi(\boldsymbol{Z}) \boldsymbol{W}_{g}=Q(x, y ; \boldsymbol{Z}) \frac{\partial}{\partial y} \tau(x, y)+R(x, y ; \boldsymbol{Z}),
$$

так что степень полинома $R$ по переменной $x$ не превосходит $[(g-1) / 3]-1$, а по переменной $y$ не превосходит 2. Введем подпространство $\mathscr{L} \subset \mathbb{C}^{g} \times \mathbb{C}^{3 g} \times \mathbb{C}^{\delta}$, выделяемое $3[(g-1) / 3]$ условиями $R(x, y ; \boldsymbol{Z}) \equiv 0$. Тогда из сравнения с $(2.1)$ получаем соотношение

$$
Q(x, y ; \boldsymbol{Z})-2 f_{g}(x, y, \boldsymbol{\lambda})=0,
$$

которое позволяет определить расслоение $\left(\mathscr{L}, \mathrm{M}_{g}, p_{\mathscr{L}}\right)$. Проекция $p_{\mathscr{L}}$ отображает точку $\boldsymbol{Z} \in \mathscr{L}$ в упорядоченный по убыванию весов набор коэффициентов положительного веса полинома $-\frac{1}{2} Q(x, y ; \boldsymbol{Z})$. Отметим, что проекция $p_{\mathscr{L}}$ является рациональным отображением.

Лемма 2.5. Пусть полиномь $\rho^{ \pm}(x, y)$ u $\tau(x, y)$ взаимно простьл в кольце $\mathbb{C}[x, y]$. Тогда они имеют $g$ совместных нулей $\left\{\left(\xi_{i}^{ \pm}, \eta_{i}^{ \pm}\right)\right\}_{i=1, \ldots, g}$.

Более того, $f_{g}\left(\xi_{i}^{ \pm}, \eta_{i}^{ \pm} ; p_{\mathscr{L}}(\boldsymbol{Z})\right)=0$ для всех $i=1, \ldots, g$. 
ДоКАЗАТЕЛЬСТВо. Полиномы $\rho^{ \pm}(x, y)$ и $\tau(x, y)$ линейны по переменной $y$. Их коэффициенты при $y^{1}$ являются полиномами по $x$ степеней $[g / 3]$ и $[(g-1) / 3]$ соответственно, а коэффициенты при $y^{0}$ имеют степени $[(2 g+1) / 3]$ и $[2 g / 3]$. Исключая $y$, получаем полином по $x$ степени $\max ([(2 g+1) / 3]+[(g-1) / 3]$, $[2 g / 3]+[g / 3])$, а это выражение при условии, что $\operatorname{gcd}(g+1,3)=1$, равно $g$. По предположению этот полином не равен нулю тождественно.

Далее, из (2.6) следует, что $\boldsymbol{W}_{g}^{T}\left(\xi_{i}^{ \pm}, \eta_{i}^{ \pm}\right) \varphi(\boldsymbol{Z}) \boldsymbol{W}_{g}\left(\xi_{i}^{ \pm}, \eta_{i}^{ \pm}\right)=0$ для всех $i=$ $1, \ldots, g$. Предположим, что $f_{g}\left(\xi, \eta ; p_{\mathscr{L}}(\boldsymbol{Z})\right) \neq 0$ для какой-нибудь точки $(\xi, \eta)$ из набора общих нулей. Тогда, поскольку $\frac{\partial}{\partial y} \tau(x, y)$ не зависит от $y$, заключаем, что $\boldsymbol{W}_{g}^{T}(\xi, y) \varphi(\boldsymbol{Z}) \boldsymbol{W}_{g}(\xi, y)=0$ для произвольного $y$. Последнее равенство возможно, только если полиномы $\rho^{ \pm}(x, y)$ и $\tau(x, y)$ имеют общий множитель $x-\xi$.

Теперь мы готовы сформулировать основной результат этого параграфа.

Tеорема 2.6. Отображение $\alpha$ из пространства $\mathscr{L}$ в пространство $g$ - $x$ симметрических степеней тригональных кривых $\mathrm{U}_{g}$, сопоставляющее набору $\boldsymbol{Z}=\left(\boldsymbol{z},\left(\boldsymbol{\gamma}_{1}, \boldsymbol{\gamma}_{2}, \boldsymbol{\gamma}_{3}\right), \Delta_{g}\right) \in \mathscr{L}$ вектор $\boldsymbol{\lambda}=p_{\mathscr{L}}(\boldsymbol{Z})$ и набор совместньх нулей взаимно простых полиномов $\tau(x, y)$ и $\rho^{+}(x, y)$, задает послойную бирачиональную эквивалентность расслоений $\alpha:\left(\mathscr{L}, \mathrm{M}_{g}, p_{\mathscr{L}}\right) \rightarrow\left(\mathrm{U}_{g}, \mathrm{M}_{g}, p_{\mathrm{U}}\right)$, тождественную на общей базе $\mathrm{M}_{g}$.

ДокАЗАтЕльство. Построим отображение $\alpha^{-1}$.

Пусть дана точка $\left(\left\{\left(\xi_{i}, \eta_{i}\right)\right\}_{i=1, \ldots, g}, \boldsymbol{\lambda}\right) \in \mathrm{U}_{g}$. В силу формулы (2.5) для решения нашей задачи достаточно найти полиномы $\tau(x, y), \chi(x, y), \rho^{+}(x, y)$ и $\rho^{-}(x, y)$.

Пусть $g=3 \ell+1$. Обозначим через $\boldsymbol{U}(x, y)$ вектор, составленный из первых $g$ компонент вектора $\boldsymbol{W}_{g}(x, y)$, и положим

$$
\begin{gathered}
\boldsymbol{t}(x, y)=\left(\boldsymbol{U}(x, y)^{T}, y x^{\ell}\right)^{T}, \quad \boldsymbol{r}(x, y)=\left(\boldsymbol{U}(x, y)^{T},-\lambda_{3 g+2} y x^{\ell}-2 x^{2 \ell+1}\right)^{T}, \\
\boldsymbol{p}(x, y)=\left(\boldsymbol{U}(x, y)^{T}, y x^{\ell}, 2 y^{2}\right)^{T} .
\end{gathered}
$$

Предположим, что $\operatorname{det}\left(\boldsymbol{U}\left(\xi_{1}, \eta_{1}\right), \ldots, \boldsymbol{U}\left(\xi_{g}, \eta_{g}\right)\right) \neq 0$. Рассмотрим целую рациональную функцию

$$
T(x, y)=\frac{\operatorname{det}\left(\boldsymbol{t}(x, y), \boldsymbol{t}\left(\xi_{1}, \eta_{1}\right), \ldots, \boldsymbol{t}\left(\xi_{g}, \eta_{g}\right)\right)}{\operatorname{det}\left(\boldsymbol{U}\left(\xi_{1}, \eta_{1}\right), \ldots, \boldsymbol{U}\left(\xi_{g}, \eta_{g}\right)\right)}=y x^{\ell}+\ldots
$$

на кривой $V$, заданной числовыми параметрами $\boldsymbol{\lambda}$. Порядок функции $T(x, y)$ равен $\operatorname{deg}\left(y x^{\ell}\right)=g+1+3 \ell=2 g$; следовательно, помимо нулей $\left\{\left(\xi_{i}, \eta_{i}\right)\right\}_{i=1, \ldots, g}$ она имеет на кривой $V$ еще $g$ нулей $\left\{\left(\widetilde{\xi}_{i}, \widetilde{\eta}_{i}\right)\right\}_{i=1, \ldots, g}$. С помощью этих двух наборов нулей зададим на кривой $V$ пару целых рациональных функций порядка $2 g+1$ :

$$
\begin{aligned}
& R_{1}(x, y)=\frac{\operatorname{det}\left(\boldsymbol{r}(x, y), \boldsymbol{r}\left(\xi_{1}, \eta_{1}\right), \ldots, \boldsymbol{r}\left(\xi_{g}, \eta_{g}\right)\right)}{\operatorname{det}\left(\boldsymbol{U}\left(\xi_{1}, \eta_{1}\right), \ldots, \boldsymbol{U}\left(\xi_{g}, \eta_{g}\right)\right)}=-2 x^{2 \ell+1}-\lambda_{3 g+2} y x^{\ell}+\ldots, \\
& R_{2}(x, y)=\frac{\operatorname{det}\left(\boldsymbol{r}(x, y), \boldsymbol{r}\left(\widetilde{\xi}_{1}, \widetilde{\eta}_{1}\right), \ldots, \boldsymbol{r}\left(\widetilde{\xi}_{g}, \widetilde{\eta}_{g}\right)\right)}{\operatorname{det}\left(\boldsymbol{U}\left(\widetilde{\xi}_{1}, \widetilde{\eta}_{1}\right), \ldots, \boldsymbol{U}\left(\widetilde{\xi}_{g}, \widetilde{\eta}_{g}\right)\right)}=-2 x^{2 \ell+1}-\lambda_{3 g+2} y x^{\ell}+\ldots
\end{aligned}
$$

Функция $R_{1}(x, y)$ имеет на кривой $g+1$ дополнительных нулей $\left\{\left(\varrho_{i}, \varsigma_{i}\right)\right\}_{i=1, \ldots, g+1}$, а функция $R_{2}(x, y)$ - дополнительные нули $\left\{\left(\varrho_{i}, \tilde{\varsigma}_{i}\right)\right\}_{i=1, \ldots, g+1}$.

Дробь $R_{1}(x, y) R_{2}(x, y) /(2 T(x, y))$ представляет иелую (все нули знаменателя сокращаются) рациональную функцию $P(x, y)$ на кривой $V$ порядка $2 g+2$, все 
нули которой нам известны. Изучим поведение функции $P(x, y)$ в окрестности точки $(\infty, \infty)$. Пусть $t$ - локальный параметр, $x=t^{-3}$ и $y=t^{-(g+1)}(1+$ $\left.\frac{1}{3} \lambda_{3 g+2} t+O\left(t^{2}\right)\right)$. Получаем $P(x, y)=t^{-(2 g+2)}\left(2+\frac{4}{3} \lambda_{3 g+2} t+O\left(t^{2}\right)\right)$. Из приведенных выражений следует, что функция $P(x, y)$ допускает полиномиальное представление вида $2 y^{2}+P_{2 g+1} x^{2 \ell+1}+\ldots$ с коэффициентом $P_{2 g+1}=0$, и, таким образом, для получения явного выражения достаточно задать какие-либо $g+1$ из ее нулей. Используя набор $\left\{\left(\varrho_{i}, \varsigma_{i}\right)\right\}_{i=1, \ldots, g+1}$, получаем

$$
P(x, y)=\frac{\operatorname{det}\left(\boldsymbol{p}(x, y), \boldsymbol{p}\left(\varrho_{1}, \varsigma_{1}\right), \ldots, \boldsymbol{p}\left(\varrho_{g+1}, \varsigma_{g+1}\right)\right)}{\operatorname{det}\left(\boldsymbol{t}\left(\varrho_{1}, \varsigma_{1}\right), \ldots, \boldsymbol{t}\left(\varrho_{g+1}, \varsigma_{g+1}\right)\right)} .
$$

Рассмотрим полином

$$
C(x, y)=P(x, y) T(x, y)-\frac{1}{2} R_{1}(x, y) R_{2}(x, y)=2 x^{\ell} y^{3}-2 x^{\ell} x^{g+1}+\ldots
$$

как функцию на кривой $V$. Эта функция имеет $4 g+2$ нулей. Приведем функцию $C(x, y)$ к нормальному виду, т. е., прибавляя кратные тригонального полинома $f_{g}(x, y ; \boldsymbol{\lambda})$, избавимся от слагаемых, содержащих $y$ в степени выше 2. Коэффициент при $y^{3}$ равен $2 \frac{\partial}{\partial y} T(x, y)$, более высокие степени $y$ отсутствуют, и потому разность $C(x, y)-2 f_{g}(x, y ; \boldsymbol{\lambda}) \frac{\partial}{\partial y} T(x, y)$ уже имеет нормальный вид. Заметим, однако, что порядок этой разности $<4 g+2$ ввиду сокращения старших степеней $x$. Таким образом, число нулей функции $C(x, y)-2 f_{g}(x, y ; \lambda) \frac{\partial}{\partial y} T(x, y)$ больше ее порядка, и, следовательно, она равна нулю тождественно.

Сравнивая тождество

$$
P(x, y) T(x, y)-\frac{1}{2} R_{1}(x, y) R_{2}(x, y) \equiv 2 f_{g}(x, y ; \boldsymbol{\lambda}) \frac{\partial}{\partial y} T(x, y)
$$

с соотношением (2.6), получаем следующие выражения для искомых полиномов (с точностью до перемены мест в паре $\rho^{+}$и $\rho^{-}$):

$$
\begin{aligned}
& \tau(x, y)=T(x, y), \quad \rho^{+}(x, y)= \\
& \chi(x, y)=\frac{1}{2}\left(P(x, y)-\frac{h_{g+1, g+2}}{2}(x, y), \quad R_{1}(x, y)+R_{2}(x, y)\right) \\
&\left.+\frac{2 h_{g+1, g+1}+h_{g+1, g+2}^{2}}{2} T(x, y)\right) .
\end{aligned}
$$

Остается выразить матричные элементы $h_{g+1, g+1}$ и $h_{g+1, g+2}$ через $\boldsymbol{\lambda}$ и коэффициенты полинома $P(x, y)$. Из формулы $(2.5)$ следует, что

$$
\begin{aligned}
\rho^{+}(x, y) & =-2 x^{2 \ell+1}+h_{g+1, g+2} y x^{\ell}+\ldots, \\
\chi(x, y) & =y^{2}+h_{g+1, g+2} x^{2 \ell+1}+h_{g+1, g+1} y x^{\ell}+\ldots,
\end{aligned}
$$

откуда получаем $h_{g+1, g+2}=-\lambda_{3 g+2}$ и $h_{g+1, g+1}=\frac{1}{6}\left(2 P_{2 g}-\lambda_{3 g+2}^{2}\right)$, где $P_{2 g}-$ коэффициент при $y x^{\ell}$ из полинома $P(x, y)$.

Случай $g=3 \ell$ рассматривается аналогично.

Далее, как и в предыдущей теореме, мы будем иметь дело с различными расслоениями над одной и той же базой $\mathrm{M}_{g}$. Формулируя утверждения о nослойньıx отображениях таких расслоений, мы будем иметь в виду только отображения, тождественные на базе, не оговаривая этого впредь явно. 


\section{§3. Универсальные пространства многообразий Якоби и Куммера}

Каждому набору $\boldsymbol{\lambda}$, определяющему тригональную кривую как множество нулей полинома (1.1), можно однозначно сопоставить решетку $\Lambda=\Lambda(\boldsymbol{\lambda})$ в $\mathbb{C}^{g}$, порожденную периодами $g$ базисных дифференциалов

$$
\mathrm{d} u_{i}(x, y ; \boldsymbol{\lambda})=\frac{w_{i}(x, y)}{\frac{\partial}{\partial y} f_{g}(x, y ; \boldsymbol{\lambda})} \mathrm{d} x, \quad i=1, \ldots, g,
$$

где $w_{i}(x, y)$, как и выше, обозначает $i$-ю координату вектора $\boldsymbol{W}(x, y)$.

ОПРЕДЕЛЕНИЕ 3.1. Универсальным пространством многообразий Якоби тригональных кривых будем называть факторпространство $\mathrm{J}_{g}=\mathbb{C}^{g} \times \mathrm{M}_{g} / \sim$, где отношение эквивалентности задано формулой $(\boldsymbol{u}, \boldsymbol{\lambda}) \sim\left(\boldsymbol{u}^{\prime}, \boldsymbol{\lambda}^{\prime}\right) \Longleftrightarrow(\boldsymbol{\lambda}=$ $\left.\boldsymbol{\lambda}^{\prime}, \boldsymbol{u}-\boldsymbol{u}^{\prime} \in \Lambda(\boldsymbol{\lambda})\right)$.

Универсальным пространством многообразий Куммера тригональных кривblx будем называть факторпространство пространства $J_{g}$ по канонической инволюции $(\boldsymbol{u}, \boldsymbol{\lambda}) \rightarrow(-\boldsymbol{u}, \boldsymbol{\lambda})$; мы будем обозначать его через $\mathrm{K}_{g}$.

ОПРЕДЕЛЕНИЕ 3.2. Универсальньм расслоением многообразий Якоби тригональньъх кривьхх будем называть тройку $\left(\mathrm{J}_{g}, \mathrm{M}_{g}, p_{\mathrm{J}}\right)$, где $p_{\mathrm{J}}(\boldsymbol{u}, \boldsymbol{\lambda})=\boldsymbol{\lambda}$.

Универсальньл расслоением многообразий Куммера тригональных кривых будем называть тройку $\left(\mathrm{K}_{g}, \mathrm{M}_{g}, p_{\mathrm{K}}\right)$, где $p_{\mathrm{K}}(\boldsymbol{u}, \boldsymbol{\lambda})=\boldsymbol{\lambda}$.

Слои этих расслоений над точкой $\boldsymbol{\lambda} \in \mathrm{M}_{g}$ называются многообразием Якоби $\operatorname{Jac}(V)$ и многообразием Куммера $\operatorname{Kum}(V)$ тригональной кривой $V(x, y)$ в модели, заданной нулями полинома $f_{g}(x, y ; \boldsymbol{\lambda})$.

С помощью дифференциалов (3.1) задается отображение Абеля $A: \mathrm{U}_{g} \rightarrow$ $\mathbb{C}^{g} \times \mathrm{M}_{g}, A\left(\left\{\left(x_{1}, y_{1}\right), \ldots,\left(x_{g}, y_{g}\right)\right\} ; \boldsymbol{\lambda}\right)=(\boldsymbol{u}, \boldsymbol{\lambda})$, где $\boldsymbol{u}=\left(u_{1}, \ldots, u_{g}\right)$ и $u_{i}=$ $\sum_{k=1}^{g} \int_{(\infty, \infty)}^{\left(x_{k}, y_{k}\right)} \mathrm{d} u_{i}(x, y ; \boldsymbol{\lambda})$. Это отображение многозначно, так как, в зависимости от выбора путей интегрирования, одному набору $\left(\left\{\left(x_{1}, y_{1}\right), \ldots,\left(x_{g}, y_{g}\right)\right\} \in\right.$ $(V)^{g}$ соответствует счетное число значений $\boldsymbol{u}_{1}, \boldsymbol{u}_{2}, \ldots$ в $\mathbb{C}^{g}$. Известно, однако, что попарные разности $\boldsymbol{u}_{i}-\boldsymbol{u}_{j}$ этих значений лежат в $\Lambda(\boldsymbol{\lambda})$. Таким образом, это отображение задает послойную бирациональную эквивалентность расслоений $A:\left(\mathrm{U}_{g}, \mathrm{M}_{g}, p_{\mathrm{U}}\right) \rightarrow\left(\mathrm{J}_{g}, \mathrm{M}_{g}, p_{\mathrm{J}}\right)$.

Непосредственно из конструкции расслоения $\left(\mathscr{L}, \mathrm{M}_{g}, p_{\mathscr{L}}\right)$ следует, что отображение $\left(\boldsymbol{z},\left(\gamma_{1}, \gamma_{2}, \gamma_{3}\right), \Delta_{g}\right) \mapsto\left(-\boldsymbol{z},\left(\gamma_{1}, \gamma_{2}, \gamma_{3}\right), \Delta_{g}\right)$ индуцирует на нем послойную инволюцию. Фактор по этой инволюции, которую мы будем называть канонической, задает расслоение $\left(\mathscr{K}, \mathrm{M}_{g}, p_{\mathscr{K}}\right)$, где $\mathscr{K}=\mathscr{T}_{g} \cap\left(\mathscr{H}_{4} \times \mathrm{M}_{g}\right)$. Проекция $p_{1}$ позволяет, согласно лемме 2.1 и следствию 2.3 , отождествить пространство $\mathscr{K}$ с матричным подпространством $p_{1}\left(\mathscr{T}_{g}\right) \cap \mathscr{H}_{4}$.

ТЕорема 3.1 (о параметризации). Имеет место послойная бираииональная эквивалентность расслоений $\mathscr{A}:\left(\mathscr{L}, \mathrm{M}_{g}, p_{\mathscr{L}}\right) \rightarrow\left(\mathrm{J}_{g}, \mathrm{M}_{g}, p_{\mathrm{J}}\right)$, коммутирующая $с$ каноническими инволючиями и индуцирующая послойную бирачиональную эквивалентность расслоений $\mathscr{B}:\left(\mathscr{K}, \mathrm{M}_{g}, p_{\mathscr{K}}\right) \rightarrow\left(\mathrm{K}_{g}, \mathrm{M}_{g}, p_{\mathrm{K}}\right)$.

ДоказАтельство. Рассмотрим композицию $\mathscr{A}=A \circ \alpha: \mathscr{L} \rightarrow \mathrm{J}_{g}$. Во-первых, заметим, что по построению

$$
p_{\mathscr{L}}\left(-\boldsymbol{z},\left(\boldsymbol{\gamma}_{1}, \boldsymbol{\gamma}_{2}, \boldsymbol{\gamma}_{3}\right), \Delta_{g}\right)=p_{\mathscr{L}}\left(\boldsymbol{z},\left(\boldsymbol{\gamma}_{1}, \boldsymbol{\gamma}_{2}, \boldsymbol{\gamma}_{3}\right), \Delta_{g}\right) .
$$


Для того чтобы показать, что отображение $\mathscr{A}$ коммутирует с инволюциями, достаточно проверить, что

$$
\mathscr{A}\left(-\boldsymbol{z},\left(\gamma_{1}, \gamma_{2}, \gamma_{3}\right), \Delta_{g}\right)+\mathscr{A}\left(\boldsymbol{z},\left(\gamma_{1}, \gamma_{2}, \gamma_{3}\right), \Delta_{g}\right) \sim(\mathbf{0}, \boldsymbol{\lambda}) \text {. }
$$

Действительно, в обозначениях леммы 2.5 включение

$$
\sum_{k=1}^{g} \int_{(\infty, \infty)}^{\left(\xi_{k}^{+}, \eta_{k}^{+}\right)} \mathrm{d} \boldsymbol{u}\left(x, y ; p_{\mathscr{L}}(\boldsymbol{Z})\right)+\sum_{k=1}^{g} \int_{(\infty, \infty)}^{\left(\xi_{k}^{-}, \eta_{k}^{-}\right)} \mathrm{d} \boldsymbol{u}\left(x, y ; p_{\mathscr{L}}(\boldsymbol{Z})\right) \in \Lambda\left(p_{\mathscr{L}}(\boldsymbol{Z})\right)
$$

следует из классической теоремы Абеля ввиду того, что множество совместных нулей пары полиномов $\left\{\tau(x, y), f_{g}\left(x, y ; p_{\mathscr{L}}(\boldsymbol{Z})\right)\right\}$ и множество совместных нулей пары $\left\{\tau(x, y), \rho^{+}(x, y) \rho^{-}(x, y)\right\}$ совпадают.

Во-вторых, заметим, что отображение $\mathscr{A}$ переводит множество ветвления накрытия $\mathscr{L} \rightarrow \mathscr{K}$, на котором все нули пары $\left\{\tau(x, y), f_{g}\left(x, y ; p_{\mathscr{L}}(\boldsymbol{Z})\right)\right\}$ двойные, в множество ветвления накрытия $\mathrm{J}_{g} \rightarrow \mathrm{K}$, т. е. в подпространство полупериодов $\left\{\left(\boldsymbol{\Omega}, p_{\mathscr{L}}(\boldsymbol{Z})\right) \mid 2 \boldsymbol{\Omega} \in \Lambda\left(p_{\mathscr{L}}(\boldsymbol{Z})\right)\right\}$, и, следовательно, индуцированное отображение $\mathscr{B}$ корректно определено.

ЗАмЕчАниЕ 3.2. Соотношение (3.2) выполняется при подходящей интерпретации периодов абелевых интегралов, т. е. решетки $\Lambda\left(p_{\mathscr{L}}(\boldsymbol{Z})\right)$, и в случае, когда полином $f_{g}\left(x, y ; p_{\mathscr{L}}(\boldsymbol{Z})\right)$ вырожден (см. обсуждение теоремы Абеля в [2]). Отображение $A$, голоморфное в невырожденном случае, становится мероморфным, а его обращение сводится к классу так называемых обобщенных задач обращения Якоби. Решение методами теории $\theta$-функций для некоторой частной постановки приведено в [7].

Слои универсальных расслоений многообразий Якоби и Куммера бирационально эквивалентны алгебраическим подмногообразиям. Используя обозначения, введенные в $\S 2$, сформулируем наш результат.

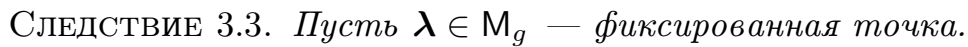

(1) Имеет место реализация многообразия $\mathrm{Jac}(V)$ как алгебраического подмногообразия в $\mathbb{C}^{4 g+\delta}$, где $\delta=2(g-3[\mathrm{~g} / 3])$. Это подмногообразие задается как множество совместных нулей системь из $2 g+3+3[(g-1) / 3]=3 g+\delta$ полиномов, порожденньх производящими функииями

$$
G_{1}(t)=\gamma_{g, 3}^{(2-\delta) / 2}\left(Q\left(t^{3}, t^{g+1} ; \boldsymbol{Z}\right)-2 f_{g}\left(t^{3}, t^{g+1} ; \boldsymbol{\lambda}\right)\right), \quad G_{2}(t)=R\left(t^{3}, t^{g+1} ; \boldsymbol{Z}\right) .
$$

(2) Имеет место реализаиия многообразия $\operatorname{Kum}(V)$ как алгебраического подмногообразия в $\mathbb{C}^{g(g+1) / 2}$. Это подмногообразие задается как множество совместных нулей системь из $(g-1) g / 2$ полиномов степени не выле 5. Эти полиномь представляют собой $5 \times 5$-минорь $(g+3) \times(g+3)$-матриць K, которая задана как анзач общего решения уравнения $\boldsymbol{W}_{g}^{T} K \boldsymbol{W}_{g}=2 r(x, y ; K) f_{g}(x, y ; \boldsymbol{\lambda})$ в пространстве матрич $\mathscr{H}$.

ДокАЗАтельство. (1) Согласно определению пространства $\mathscr{L}$, образ отображения $\mathscr{A}^{-1}$ в $\mathbb{C}^{4 g+\delta}$ задан условием $R(x, y ; \boldsymbol{Z})=0$ для всех $(x, y) \in \mathbb{C}^{2}$, откуда получаем производящую функцию $G_{2}(t, \boldsymbol{Z})$. При заданном значении $\boldsymbol{\lambda}$ соотношение (2.7) порождает уравнения ограничения на слой в расслоении $\left(\mathscr{L}, \mathrm{M}_{g}, p_{\mathscr{L}}\right)$.

(2) Произвольная матрица $K \in \mathscr{T}_{g}$ по определению имеет $g(g+1) / 2+3 g+\delta$ параметров. При заданном значении $\boldsymbol{\lambda} \in \mathbb{C}^{2 g+3}$ соотношение (2.1) налагает на 
эти параметры $2 g+3+3[(g-1) / 3]$ линейных условий (действительно, формально это соотношение задает некоторую целую рациональную нормального вида функцию порядка $3 g+3+3[(g-1) / 3]$, тождественно равную нулю на кривой), которые выделяют слой $p_{\mathscr{T}}^{-1}(\boldsymbol{\lambda}) \subset \mathscr{T}_{g}$. Таким образом, общее решение уравнения $\boldsymbol{W}_{g}^{T} K \boldsymbol{W}_{g}=2 r(x, y ; K) f_{g}(x, y ; \boldsymbol{\lambda})$ в пространстве матриц $\mathscr{H}$, т. е. точка $K \in p_{\mathscr{T}}^{-1}(\boldsymbol{\lambda})$, имеет $g(g+1) / 2$ параметров. Из указанного выше отождествления $\mathscr{K}$ с пересечением $p_{1}\left(\mathscr{T}_{g}\right) \cap \mathscr{H}_{4}$ и леммы 2.1 следует, что отображение $\mathscr{B}^{-1}$ переводит слой расслоения $\left(\mathrm{K}_{g}, \mathrm{M}_{g}, p_{\mathrm{K}}\right)$ в пространство матриц $\left\{K \in p_{\mathscr{T}}^{-1}(\boldsymbol{\lambda}) \mid \operatorname{rank} K \leqslant 4\right\}$.

ПримеР 3.4. Пусть $g=3$. Тогда

$$
\boldsymbol{W}_{3}(x, y)=\left(1, x, y, x^{2}, y x, y^{2}\right)^{T} \quad \text { и } \quad R(x, y ; \boldsymbol{Z})=0 .
$$

Тригональная кривая $V(x, y)$ определяется полиномом

$$
\begin{aligned}
f_{3}(x, y ; \boldsymbol{\lambda})=y^{3}-\left(\lambda_{11} x+\lambda_{8}\right) y^{2}-\left(\lambda_{10} x^{2}+\right. & \left.\lambda_{7} x+\lambda_{4}\right) y \\
& -\left(x^{4}+\lambda_{9} x^{3}+\lambda_{6} x^{2}+\lambda_{3} x+\lambda_{0}\right) .
\end{aligned}
$$

Многообразие Якоби $\operatorname{Jac}(V)$ выделяется в пространстве $\mathbb{C}^{12}$ с координатами $\left(z_{i},\left(\gamma_{i, 1}, \gamma_{i, 2}, \gamma_{i, 3}\right)\right), i=1,2,3$, системой из 9 уравнений

$$
\begin{aligned}
& 4 \gamma_{3,3} \lambda_{0}+z_{1}^{2}+4 \gamma_{1,3}^{2} \gamma_{3,3}+4 \gamma_{1,3} \gamma_{1,1}-\gamma_{1,2}^{2}=0, \\
& 2 \gamma_{3,3} \lambda_{3}+z_{1} z_{2}+4 \gamma_{1,3} \gamma_{2,3} \gamma_{3,3}+2 \gamma_{2,3} \gamma_{1,1}+2 \gamma_{1,3} \gamma_{2,1}-\gamma_{1,2} \gamma_{2,2}=0, \\
& 2 \gamma_{3,3} \lambda_{4}+z_{1} z_{3}+4 \gamma_{1,3} \gamma_{3,3}^{2}+2 \gamma_{3,3} \gamma_{1,3}+2 \gamma_{1,3} \gamma_{3,1}-\gamma_{1,2} \gamma_{3,2}=0, \\
& 4 \gamma_{3,3} \lambda_{6}+z_{2}^{2}+4 \gamma_{2,3}^{2} \gamma_{3,3}+4 \gamma_{1,1}+4 \gamma_{2,3} \gamma_{2,1}-\gamma_{2,2}^{2}=0, \\
& 2 \gamma_{3,3} \lambda_{7}+z_{2} z_{3}+4 \gamma_{2,3} \gamma_{3,3}^{2}+2 \gamma_{3,3} \gamma_{2,1}+2 \gamma_{2,3} \gamma_{3,1}+2 \gamma_{1,2}-\gamma_{2,2} \gamma_{3,2}=0, \\
& 4 \gamma_{3,3} \lambda_{8}+z_{3}^{2}+4 \gamma_{1,3}+4 \gamma_{3,3}^{3}+4 \gamma_{3,3} \gamma_{3,1}-\gamma_{3,2}^{2}=0, \\
& \gamma_{3,3} \lambda_{9}+\gamma_{2,1}=0, \quad \gamma_{3,3} \lambda_{10}+\gamma_{2,2}+\gamma_{3,1}=0, \quad \gamma_{3,3} \lambda_{11}+\gamma_{2,3}+\gamma_{3,2}=0 .
\end{aligned}
$$

Для описания многообразия Куммера $\operatorname{Kum}(V)$ в пространстве $\mathbb{C}^{6}$ с координатами $\left(v_{i}\right), i=2,3,4,6,7,8$ (индекс у координаты равен ее весу в принятой градуировке), используется следующий анзац общего решения $K$ уравнения $\boldsymbol{W}_{3}^{T} K \boldsymbol{W}_{3}=2 r(x, y ; K) f_{3}(x, y ; \boldsymbol{\lambda})$ в классе симметрических матриц $\mathscr{H}$ при заданном значении вектора $\boldsymbol{\lambda}$ :

$$
K=\left(\begin{array}{cccccc}
-2 \lambda_{0} v_{2} & -\lambda_{3} v_{2} & -\lambda_{4} v_{2} & v_{8} & v_{7} & v_{6} \\
-\lambda_{3} v_{2} & -2 v_{8}-2 \lambda_{6} v_{2} & -v_{7}-\lambda_{7} v_{2} & -\lambda_{9} v_{2} & v_{4} & v_{3} \\
-\lambda_{4} v_{2} & -v_{7}-\lambda_{7} v_{2} & -2 v_{6}-2 \lambda_{8} v_{2} & -v_{4}-\lambda_{10} v_{2} & -v_{3}-\lambda_{11} v_{2} & v_{2} \\
v_{8} & -\lambda_{9} v_{2} & -v_{4}-\lambda_{10} v_{2} & -2 v_{2} & 0 & 1 \\
v_{7} & v_{4} & -v_{3}-\lambda_{11} v_{2} & 0 & -2 & 0 \\
v_{6} & v_{3} & v_{2} & 1 & 0 & 0
\end{array}\right) .
$$

Уравнения, выделяющие $\operatorname{Kum}(V)$, находятся из условия $\operatorname{rank} K \leqslant 4$, что сводится к трем уравнениям, которые получаются приравниванием нулю алгебраических дополнений элементов $K_{1,1}, K_{1,2}$ и $K_{2,2}$.

ЗАмечАниЕ 3.5. Можно показать, что система из $g+\delta-3$ полиномиальных уравнений, порожденных производящей функцией $G_{2}(t)$, имеет рациональное 
решение ${ }^{1}$. Используя это решение, можно на основании следствия 3.3 прийти к реализации многообразия Якоби в пространстве $\mathbb{C}^{3 g+3}$ как алгебраического подмногообразия. Однако ввиду того, что степени полиномов, порожденных функцией $G_{1}(t)$, не превосходят 4 , а степени полиномов, порожденных функцией $G_{2}(t)$, растут линейно по $g$, степени уравнений в такой реализации растут полиномиально по $g$.

Применим к матрице $K$ из п. (2) следствия 3.3 формулу (2.3). Получаем, что существует вектор $\boldsymbol{q}=\left(q_{1}, \ldots, q_{g}\right)^{T}$, такой, что

$$
q_{i} q_{j}+\frac{1}{2} \operatorname{det} K\left[\begin{array}{cccc}
i & g+1 & g+2 & g+3 \\
j & g+1 & g+2 & g+3
\end{array}\right]=0, \quad i, j \in\{1, \ldots, g\},
$$

где $K\left[\begin{array}{lll}i & \ldots & k \\ j & \ldots & l\end{array}\right]$ обозначает подматрицу матрицы $K$, составленную из элементов, находящихся на пересечении строк $(i, \ldots, k)$ и столбцов $(j, \ldots, l)$. Очевидно, найдется такой набор $\boldsymbol{Z}=\left(\boldsymbol{z},\left(\boldsymbol{\gamma}_{1}, \boldsymbol{\gamma}_{2}, \boldsymbol{\gamma}_{3}\right), \Delta_{g}\right)$ из п. (1), что $\boldsymbol{q}=\boldsymbol{z}$ удовлетворяет этим уравнениям. С другой стороны, имеем

СледСтвиЕ 3.6. Имеет место реализаиия многообразия Якоби тригональной кривой как множества совместных нулей системь из $g(g+1) / 2$ полиномов степени не выле 4 вида (3.3) в пространстве $\mathbb{C}^{g(g+3) / 2}$.

\section{$\S 4$. Униформизация многообразий Якоби}

Построенные выше модели пространств $\mathrm{J}_{g}$ допускают явную униформизацию средствами теории абелевых функций. Для простоты изложения ниже мы ограничимся рассмотрением тригональных полиномов вида (1.1) с нулевым коэффициентом при $y^{2}$. Это не приведет к ограничению общности, поскольку к такой форме можно перейти от общего полинома заменой $(x, y) \mapsto$ $\left(x, y+\frac{1}{3} \sum_{j=0}^{[(g+1) / 3]} \lambda_{2 g+2+3 j} x^{j}\right)$ и последующим переобозначением параметров.

В первой главе работы авторов [3] построена модификация стандартной $\theta$-функции, которая задает отображение $\sigma: \mathbb{C}^{g} \times \mathrm{M}_{g} \rightarrow \mathbb{C}$, являясь инвариантной относительно модулярных преобразований, т. е. ее значения, в отличие от значений $\theta$-функции, не зависят от выбора образующих решетки $\Lambda(\boldsymbol{\lambda})$. Символ $\sigma$ для таких функций ввел Клейн [10], подчеркивая аналогию с эллиптической $\sigma$-функцией Вейерштрасса. Как показано в [3], производные порядка > 1 от логарифма $\sigma$-функции являются абелевьли и одновременно модулярно инвариантныли функциями, т.е. мероморфными функциями, областью определения которых служит пространство $\mathrm{J}_{g}$. Для этих логарифмических производных $\sigma$-функции приняты следующие обозначения:

$$
\wp_{i_{1}, i_{2}, \ldots, i_{k}}(\boldsymbol{u}, \boldsymbol{\lambda})=-\frac{\partial^{k} \log \sigma(\boldsymbol{u}, \boldsymbol{\lambda})}{\partial u_{i_{1}} \cdots \partial u_{i_{k}}}, \quad k \geqslant 2 .
$$

Отметим свойство четности ю-функций: $\wp_{i_{1}, \ldots, i_{k}}(-\boldsymbol{u}, \boldsymbol{\lambda})=(-1)^{k} \wp_{i_{1}, \ldots, i_{k}}(\boldsymbol{u}, \boldsymbol{\lambda})$.

Приведенные далее результаты опираются на следующее соотношение, аналог которого имеет место и для $\theta$-функций [7], однако существенная для нас

1Это непосредственно следует, например, из рациональности универсальных пространств симметрических степеней алгебраических кривых [5]. 
явная зависимость правой части этого соотношения от параметров модели кривой может быть получена только для модулярно инвариантной левой части:

$$
\begin{aligned}
\sum_{i, j=1}^{g} \wp_{i, j}\left(\boldsymbol{v}-\int_{(\infty, \infty)}^{(\xi, \eta)} \mathrm{d} \boldsymbol{u}, \boldsymbol{\lambda}\right) \mathrm{d} u_{i}(\xi, \eta ; \boldsymbol{\lambda}) \mathrm{d} u_{j}(x, y ; \boldsymbol{\lambda}) & \\
& =\frac{\mathscr{F}((\xi, \eta),(x, y) ; \boldsymbol{\lambda}) \mathrm{d} \xi \mathrm{d} x}{(\xi-x)^{2} \frac{\partial}{\partial y} f_{g}(x, y ; \boldsymbol{\lambda}) \frac{\partial}{\partial \eta} f_{g}(\xi, \eta ; \boldsymbol{\lambda})}
\end{aligned}
$$

где $(x, y)$ принадлежит любому из наборов $\left\{\left\{\left(x_{1}, y_{1}\right), \ldots,\left(x_{g}, y_{g}\right)\right\} \in(V)^{g}\right.$ $\left.A\left(\left\{\left(x_{1}, y_{1}\right), \ldots,\left(x_{g}, y_{g}\right)\right\} ; \boldsymbol{\lambda}\right) \sim(\boldsymbol{v}, \boldsymbol{\lambda})\right\}$, а $(\xi, \eta)$ - произвольная точка кривой $V$. В рассматриваемом случае тригональных кривых для $\mathscr{F}((\xi, \eta),(x, y) ; \boldsymbol{\lambda})$ имеет место выражение

$$
\begin{aligned}
\mathscr{F}((\xi, \eta),(x, y) ; \boldsymbol{\lambda})=3 y^{2} \eta^{2}-y^{2} P_{1}(\xi)-\eta^{2} P_{1}(x)+y \eta \Psi_{2}\left(P_{1} ; x, \xi\right) \\
+\eta \Psi_{3}\left(P_{0} ; x, \xi\right)+y \Psi_{3}\left(P_{0} ; \xi, x\right)+\frac{1}{2} \Psi_{2}\left(P_{1}^{2} ; x, \xi\right),
\end{aligned}
$$

где $\Psi_{r}(q ; x, \xi)=\sum_{k \geqslant 0} q_{k} x^{k-[k / r]-1} \xi^{[k / r]}((r[k / r]+r-k) x+(k-r[k / r]) \xi)$ для любого полинома $q(t)=\sum_{k \geqslant 0} q_{k} t^{k}$, а полиномы $P_{1}(x)=\sum_{j=0}^{[(2 g+2) / 3]} \lambda_{g+1+3 j} x^{j}$ и $P_{0}(x)=x^{g+1}+\sum_{j=0}^{g} \lambda_{3 j} x^{j}$ - коэффициенты при степенях переменной $y$ тригонального полинома. Обратим внимание, что $\Psi_{r}(q ; x, x)=r q(x)$ и, таким образом,

$$
\mathscr{F}((x, y),(x, y) ; \boldsymbol{\lambda})=\left(\frac{\partial}{\partial y} f_{g}(x, y ; \boldsymbol{\lambda})\right)^{2}-f_{g}(x, y ; \boldsymbol{\lambda}) \frac{\partial^{2}}{\partial y^{2}} f_{g}(x, y ; \boldsymbol{\lambda}) .
$$

Положим $\wp_{k}^{T}=\left(\wp_{i, k}(\boldsymbol{v}, \boldsymbol{\lambda})\right), i=1, \ldots, g$, и $\frac{\partial}{\partial v_{g}}(\ldots)=(\ldots)^{\prime}$.

TеоремА 4.1. Зададим отображение $\pi: J_{g} \rightarrow \mathbb{C}^{4 g+\delta}$ формулами

$$
\begin{gathered}
\boldsymbol{z}=\wp_{g}^{\prime}, \quad \gamma_{3}=-\wp_{g}, \quad \boldsymbol{\gamma}_{2}=\wp_{g-1}, \\
\boldsymbol{\gamma}_{1}= \begin{cases}\frac{1}{3}\left(\wp_{g}^{\prime \prime}-\left(6 \wp_{g, g}(\boldsymbol{v}, \boldsymbol{\lambda})+\lambda_{3 g+1}\right) \wp_{g}+\boldsymbol{L}_{g}\right), & g=3 \ell, \\
\frac{1}{3}\left(\wp_{g}^{\prime \prime}-\left(6 \wp_{g, g}(\boldsymbol{v}, \boldsymbol{\lambda})+\frac{1}{2} \lambda_{3 g+2}^{2}\right) \wp_{g}+\lambda_{3 g+2} \wp_{g-1}+\boldsymbol{L}_{g}\right), & g=3 \ell+1,\end{cases}
\end{gathered}
$$

где $\boldsymbol{L}_{g}^{T}=\left(\lambda_{g+1+i} \delta_{i, 3[i / 3]}\right), i=0, \ldots, g-1$ (здесь $\delta_{i, j}-$ символ Кронекера $), u$

$$
h_{g+1, g+1}=2 \wp_{g, g}(\boldsymbol{v}, \boldsymbol{\lambda}), \quad h_{g+1, g+2}= \begin{cases}0, & g=3 \ell, \\ -\lambda_{3 g+2}, & g=3 \ell+1 .\end{cases}
$$

Мероморфное отображение $\pi$ униформизует подпространство $\mathscr{L} \subset \mathbb{C}^{4 g+\delta}$ $u$ определяет послойное отображение расслоений $\left(\mathrm{J}_{g}, \mathrm{M}_{g}, p_{\mathrm{J}}\right) \rightarrow\left(\mathscr{L}, \mathrm{M}_{g}, p_{\mathscr{L}}\right)$, обратное $\kappa$ отображению $\mathscr{A}$ из теоремь 3.1.

ДокАЗАтЕЛЬство. Пусть $\sigma(\boldsymbol{v}, \boldsymbol{\lambda}) \neq 0$ и $\boldsymbol{v}$ - не полупериод, т. е. $2 \boldsymbol{v} \notin \Lambda(\boldsymbol{\lambda})$. Тогда соотношение (4.1) эквивалентно следующему утверждению: целая рациональная функция порядка $2 g+4$

$$
\begin{aligned}
\Phi^{ \pm}(x, y, \boldsymbol{v})=\mathscr{F}((\xi, \eta), & (x, y) ; \boldsymbol{\lambda}) \\
& -(x-\xi)^{2} \sum_{i, j=1}^{g} \wp_{i, j}\left(\boldsymbol{v} \mp \int_{(\infty, \infty)}^{(\xi, \eta)} \mathrm{d} \boldsymbol{u}, \boldsymbol{\lambda}\right) w_{i}(\xi, \eta) w_{j}(x, y)
\end{aligned}
$$


имеет на кривой $V(x, y)$ в точности $g$ нулей $\left\{\left(x_{1}^{ \pm}, y_{1}^{ \pm}\right), \ldots,\left(x_{g}^{ \pm}, y_{g}^{ \pm}\right)\right\}$, не зависящих от произвольной точки $(\xi, \eta) \in V$.

Рассмотрим случай $g=3 \ell$. Используя параметризацию $\xi=t^{-3}$ и $\eta=$ $t^{-(g+1)}\left(1+\frac{1}{3} \lambda_{3 g+1} t^{2}+\ldots\right)$, исследуем поведение функции $\Phi^{ \pm}(x, y)$ в окрестности $(\xi, \eta)=(\infty, \infty)$. Имеем

причем

$$
\Phi^{ \pm}(x, y, \boldsymbol{v})=t^{-2 g-4} \sum_{i \geqslant 0} \Phi_{i}^{ \pm}(x, y, \boldsymbol{v}) t^{i},
$$

$$
\begin{gathered}
\Phi_{0}^{ \pm}(x, y, \boldsymbol{v})=\Phi_{0}(x, y, \boldsymbol{v})=w_{g+1}(x, y)-\wp_{g}^{T} \boldsymbol{U}(x, y), \\
\Phi_{1}^{ \pm}(x, y, \boldsymbol{v})=2 w_{g+2}(x, y)-\left(\wp_{g-1} \pm \wp_{g}^{\prime}\right)^{T} \boldsymbol{U}(x, y), \\
\Phi_{2}^{ \pm}(x, y, \boldsymbol{v})=\frac{\partial}{\partial y} f_{g}(x, y ; \boldsymbol{\lambda})-\frac{1}{2}\left(\wp_{g}^{\prime \prime} \pm \wp_{g-1}^{\prime}\right)^{T} \boldsymbol{U}(x, y)+\frac{\lambda_{3 g+1}}{3} \Phi_{0}(x, y \boldsymbol{v}),
\end{gathered}
$$

где, как и выше, $w_{i}(x, y)$ обозначает $i$-ю координату вектора $\boldsymbol{W}_{g}(x, y)$ и $\boldsymbol{U}(x, y)$ $=\left(w_{1}(x, y), \ldots, w_{g}(x, y)\right)^{T}$.

Функции $\left\{\Phi_{0}(x, y, \boldsymbol{v}), \Phi_{1}^{ \pm}(x, y, \boldsymbol{v}), \ldots\right\}$ одновременно обращаются в нуль на множествах точек $\left\{\left(x_{1}^{ \pm}, y_{1}^{ \pm}\right), \ldots,\left(x_{g}^{ \pm}, y_{g}^{ \pm}\right)\right\} \in(V)^{g}$. Согласно теоремам 2.6 и 3.1, наборы $\left( \pm \boldsymbol{z},\left(\boldsymbol{\gamma}_{1}, \boldsymbol{\gamma}_{2}, \boldsymbol{\gamma}_{3}\right)\right)=\alpha^{-1}\left(\left\{\left(x_{j}^{ \pm}, y_{j}^{ \pm}\right)\right\}, \boldsymbol{\lambda}\right)$ принадлежат $\mathscr{L}$. Отметим, что условие $\sigma(\boldsymbol{v}, \boldsymbol{\lambda}) \neq 0$ эквивалентно невырожденности матриц $\left(\boldsymbol{U}\left(x_{i}^{ \pm}, y_{i}^{ \pm}\right)\right), i=$ $1, \ldots, g$.

Отождествляя множества совместных нулей пар полиномов $\Phi_{0}(x, y, \boldsymbol{v})$ и $\Phi_{1}^{ \pm}(x, y, \boldsymbol{v})$ с множествами нулей $\left\{\left(\xi_{j}^{ \pm}, \eta_{j}^{ \pm}\right)\right\}$из леммы 2.5, получаем

$$
\tau(x, y)=\Phi_{0}(x, y, \boldsymbol{v}) \quad \text { и } \quad \rho^{ \pm}(x, y)=-\Phi_{1}^{ \pm}(x, y, \boldsymbol{v}),
$$

откуда следует (4.2). Остается выразить вектор $\gamma_{1}$ через ю-функции и $\boldsymbol{\lambda}$. Используем тождество (см. (2.1) и (2.6))

$$
\begin{gathered}
\left(2 y^{2}-4 \wp_{g, g}(\boldsymbol{v}, \boldsymbol{\lambda}) w_{g+1}(x, y)+2 \boldsymbol{U}(x, y)^{T} \boldsymbol{\gamma}_{1}-2 \wp_{g, g}(\boldsymbol{v}, \boldsymbol{\lambda}) \Phi_{0}(x, y, \boldsymbol{v})\right) \Phi_{0}(x, y, \boldsymbol{v}) \\
-\frac{1}{2} \Phi_{1}^{-}(x, y, \boldsymbol{v}) \Phi_{1}^{+}(x, y, \boldsymbol{v})-2 f_{g}(x, y ; \boldsymbol{\lambda}) \frac{\partial}{\partial y} \Phi_{0}(x, y, \boldsymbol{v})=0,
\end{gathered}
$$

которое выполняется для всех $(x, y) \in \mathbb{C}^{2}$. Продифференцируем (4.4) по $v_{g}$ и подставим $(x, y)=\left(x_{k}^{+}, y_{k}^{+}\right)$для некоторого $k \in\{1, \ldots, g\}$. Получаем

$\Phi_{1}^{-}\left(x_{k}^{+}, y_{k}^{+}, \boldsymbol{v}\right)\left\{\left(y_{k}^{+}\right)^{2}+\boldsymbol{U}\left(x_{k}^{+}, y_{k}^{+}\right)^{T}\left(\boldsymbol{\gamma}_{1}+2 \wp_{g, g}(\boldsymbol{v}, \boldsymbol{\lambda}) \wp_{g}\right)+\frac{1}{2} \frac{\partial}{\partial v_{g}} \Phi_{1}^{+}\left(x_{k}^{+}, y_{k}^{+}, \boldsymbol{v}\right)\right\}=0$,

где мы использовали тождество

$$
\frac{\partial}{\partial v_{g}} \Phi_{0}\left(x_{k}^{+}, y_{k}^{+}, \boldsymbol{v}\right)=\frac{1}{2}\left(\Phi_{1}^{+}\left(x_{k}^{+}, y_{k}^{+}, \boldsymbol{v}\right)-\Phi_{1}^{-}\left(x_{k}^{+}, y_{k}^{+}, \boldsymbol{v}\right)\right)=-\frac{1}{2} \Phi_{1}^{-}\left(x_{k}^{+}, y_{k}^{+}, \boldsymbol{v}\right) .
$$

Таким образом, поскольку $\Phi_{1}^{-}\left(x_{k}^{+}, y_{k}^{+}, \boldsymbol{v}\right) \neq 0$, если $\boldsymbol{v}$ - не полупериод,

$$
-\left(y_{k}^{+}\right)^{2}=\boldsymbol{U}\left(x_{k}^{+}, y_{k}^{+}\right)^{T}\left\{\boldsymbol{\gamma}_{1}-\frac{1}{2} \wp_{g}^{\prime \prime}-\frac{1}{2} \wp_{g-1}^{\prime}+2 \wp_{g, g}(\boldsymbol{v}, \boldsymbol{\lambda}) \wp_{g}\right\} ;
$$

с другой стороны, из равенства $\Phi_{2}^{+}\left(x_{k}^{+}, y_{k}^{+}, \boldsymbol{v}\right)=0$ следует

$$
-\left(y_{k}^{+}\right)^{2}=\boldsymbol{U}\left(x_{k}^{+}, y_{k}^{+}\right)^{T}\left\{-\frac{1}{6} \wp_{g}^{\prime \prime}-\frac{1}{2} \wp_{g-1}^{\prime}\right\}+\frac{1}{3} \sum_{j=0}^{[(2 g+2) / 3]} \lambda_{g+1+3 j} x^{j},
$$


и ввиду невырожденности матрицы $\left(\boldsymbol{U}\left(x_{k}^{+}, y_{k}^{+}\right)\right)_{k=1, \ldots, g}$ мы приходим к (4.3).

Случай $g=3 \ell+1$ рассматривается аналогично.

\section{5. Семейство нелинейных дифференциальных уравнений, интегрируемых в тригональных ю-функциях}

В свете результатов теоремы 4.1 производящие функции $G_{1}(t)$ и $G_{2}(t)$, введенные в п. (1) следствия 3.3, получают новую интерпретацию. Пусть вектор $\boldsymbol{Z}$ задан формулами (4.2) и (4.3); тогда полиномы, порожденные функциями $G_{1}(t)$ и $G_{2}(t)$, представляют собой дифференииальные соотношения, которым

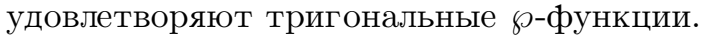

С другой стороны, задавая вектор $\boldsymbol{Z}$ формулами $\gamma_{1}=\frac{1}{3}\left(\boldsymbol{u}^{\prime \prime}-\left(6 u_{g}+\delta_{g, 3[g / 3]} \lambda_{3 g+1}+\delta_{g, 1+3[g / 3]} \frac{1}{2} \lambda_{3 g+2}^{2}\right) \boldsymbol{u}+\delta_{g, 1+3[g / 3]} \lambda_{3 g+2} \boldsymbol{q}+\boldsymbol{L}_{g}\right)$,

$\gamma_{2}=\boldsymbol{q}, \quad \gamma_{3}=-\boldsymbol{u}, \quad \boldsymbol{z}=\boldsymbol{u}^{\prime}, \quad h_{g+1, g+1}=2 u_{g}, \quad h_{g+1, g+2}=-\delta_{g, 1+3[g / 3]} \lambda_{3 g+2}$,

получаем с помощью производящих функций $G_{1}(t)$ и $G_{2}(t)$ набор дифференциальных полиномов $S_{\boldsymbol{\lambda}}^{0}=S_{\boldsymbol{\lambda}}\left(\boldsymbol{q}, \boldsymbol{u}, \boldsymbol{u}^{\prime}, \boldsymbol{u}^{\prime \prime}\right)$. Дополним набор $S_{\boldsymbol{\lambda}}^{0}$ набором линейных выражений

$$
S^{1}=\left\{q_{i}^{\prime}-\frac{\partial}{\partial v_{g-1}} u_{i} ; \frac{\partial}{\partial v_{i}} u_{k}-\frac{\partial}{\partial v_{k}} u_{i}, \frac{\partial}{\partial v_{i}} q_{k}-\frac{\partial}{\partial v_{k}} q_{i}\right\}, \quad i, k=1, \ldots, g, i>k,
$$

до набора

$$
\widehat{S}_{\boldsymbol{\lambda}}=S_{\boldsymbol{\lambda}}^{0} \cup S^{1}=\widehat{S}_{\boldsymbol{\lambda}}\left(\boldsymbol{q}, \boldsymbol{u}, \frac{\partial}{\partial v_{1}} \boldsymbol{q}, \ldots, \boldsymbol{q}^{\prime}=\frac{\partial}{\partial v_{g}} \boldsymbol{q}, \frac{\partial}{\partial v_{1}} \boldsymbol{u}, \ldots, \boldsymbol{u}^{\prime}=\frac{\partial}{\partial v_{g}} \boldsymbol{u}, \boldsymbol{u}^{\prime \prime}\right) .
$$

ПРЕДЛОЖЕНИЕ 5.1. Система $\widehat{S}_{\boldsymbol{\lambda}}=0$ нелинейных дифференциальньх уравнений второго порядка относительно функиий $\boldsymbol{q}$ и $\boldsymbol{u}$ допускает решение в тригональных ю-функииях $\boldsymbol{q}=\wp_{g-1} u \boldsymbol{u}=\wp_{g}$.

Введем градуировку для независимых переменных $\boldsymbol{v}^{T}=\left(v_{1}, \ldots, v_{g}\right)$ по правилу $\operatorname{deg} v_{i}=\operatorname{deg} w_{i}-2 g+1$, так что $\operatorname{deg} v_{g}=-1, \operatorname{deg} v_{g-1}=-2$ и т. д. (заметим, что последовательность - $\operatorname{deg} \boldsymbol{v}$ образует последовательность Вейерштрасса, порожденную парой взаимно простых чисел $(3, g+1)$; см. [4]). Для функций $\boldsymbol{q}$ и $\boldsymbol{u}$ возьмем веса, которые следуют из принятого в $\S 2$ соглашения о весах координат векторов $\gamma_{2}$ и $\gamma_{3}$, и, таким образом, $\operatorname{deg} u_{g}=2, \operatorname{deg} u_{g-1}=\operatorname{deg} q_{g}=3$ и т.д. В этой градуировке элементы набора $\widehat{S}_{\boldsymbol{\lambda}}$ являются однородными дифференциальными полиномами. Рассмотрим подробнее полиномы младших весов.

Производящие функции $G_{1}(t)$ и $G_{2}(t)$ порождают по одному полиному каждого веса. При этом функция $G_{2}(t)$ порождает полиномы веса не менее $2 g-$ $3[(g-1) / 3]=g+3-\delta$. Таким образом, элементы наименьших весов 3 и 4 в наборе $S_{\lambda}^{0}$ порождены функцией $G_{1}(t)$. А именно, имеется единственный полином $\psi_{3}=$ $q_{g}-u_{g-1}$ веса 3 и единственный полином веса 4

$$
\psi_{4}=u_{g}^{\prime \prime}-6 u_{g}^{2}+3 q_{g-1}-a q_{g}-b u_{g}-c,
$$

где

$$
\{a, b, c\}=\left\{2 \delta_{g, 1+3[g / 3]} \lambda_{3 g+2}, 4 \delta_{g, 3[g / 3]} \lambda_{3 g+1}+\delta_{g, 1+3[g / 3]} \lambda_{3 g+2}^{2}, 2 \delta_{g, 1+3[g / 3]} \lambda_{3 g-1}\right\} .
$$

Положим $v_{g}=\xi, v_{g-1}=\eta$ и $u_{g}=U / 2$, а частные производные $U$ по $\xi$ и $\eta$ будем обозначать индексами, например $\partial U / \partial \xi=U_{\xi}, \partial^{2} U / \partial \xi^{2}=U_{\xi \xi}$ и т. п. 
Из системы уравнений $\left(\psi_{3}, \psi_{4}, S^{1}\right)=0$ следует дифференциальное уравнение относительно $U$ вида

$$
3 U_{\eta \eta}+\left(U_{\xi \xi \xi}-6 U_{\xi} U\right)_{\xi}-a U_{\xi \eta}-b U_{\xi \xi}=0
$$

т.е. хорошо известное уравнение Буссинеска. По предложению 5.1 решением уравнения (5.1) является функция $U=2 \wp_{g, g}\left(u_{1}, \ldots, \eta, \xi, \boldsymbol{\lambda}\right)$, построенная по модели тригональной кривой, заданной уравнением

$$
y^{3}-y\left(\sum_{j=0}^{[(2 g+2) / 3]} \lambda_{g+1+3 j} x^{j}\right)-\left(x^{g+1}+\sum_{j=0}^{g} \lambda_{3 j} x^{j}\right)=0 .
$$

Сравним полученный результат с аналогичным результатом из гиперэллиптической теории [3]. Гиперэллиптическая функция $2 \wp_{g, g}\left(u_{1}, \ldots, \eta, \xi, \boldsymbol{\lambda}\right)$, построенная по модели гиперэллиптической кривой

$$
y^{2}-4 x^{2 g+1}-\sum_{j=0}^{2 g-1} \lambda_{2 j} x^{j}=0,
$$

является решением иерархии Кортевега-де Фриза и, в частности, классического уравнения КдФ

$$
2 U_{\eta}-U_{\xi \xi \xi}+6 U U_{\xi}=0 .
$$

При некоторых ограничениях на значения параметров $\boldsymbol{\lambda}$ гиперэллиптическая функция $2 \wp_{1,1}$ веса $4 g-2$ является решением уравнения Кадомиева-Петвиашвили

$$
4 U_{\xi \zeta}-3 U_{\eta \eta}-\left(U_{\xi \xi \xi}-6 U_{\xi} U\right)_{\xi}=0,
$$

а с помощью функции $\wp_{1, g}$ веса $2 g-2$ строится решение уравнения sine-Gordon.

В обоих случаях (5.1) и (5.2) решение $U=2 \wp_{g, g}$ - это функция веса 2 , наи-

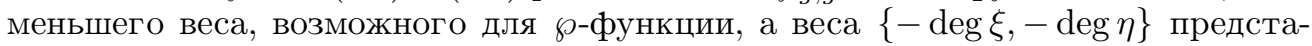
вляют собой начальные отрезки соответствующих последовательностей Вейерштрасса: $\{1,2\}$ для $(5.1)$ и $\{1,3\}$ для (5.2). При этом правые части (5.1) и $(5.2)$ получены (кратным) дифференцированием по $\xi$ полинома веса 4 , которому отвечает соотношение $\wp_{g, g, g, g}-6 \wp_{g, g}^{2}+\cdots=0$.

Элементы веса $>4$ из семейства $\widehat{S}_{\boldsymbol{\lambda}}$ после кратного дифференцирования приводят к системе нелинейных дифференциальных уравнений относительно функции $U$ - иерархии Буссинеска. Системы дифференциальных уравнений, которым удовлетворяют ю-функции старших весов, образуют семейства, не сводящиеся к иерархии Буссинеска. Детально этот круг вопросов будет разобран в наших ближайших публикациях.

ПРЕДЛОЖЕНИЕ 5.2. Для того итобъ функиия $U=2 \wp_{g, g}\left(u_{1}, \ldots, \zeta, \eta, \xi, \boldsymbol{\lambda}\right)$ веса 2, построенная по $(n, s)$-модели кривой $y^{n}-x^{s}+\cdots=0$, бьла решением уравнения (5.3), необходимо, чтобы число листов модели $n$ было не меньше 4.

ДокАЗАтЕЛьство. Действительно, применим масштабное преобразование $(U, \xi, \eta, \zeta) \mapsto\left(t^{2} U, t^{-\alpha_{1}} \xi, t^{-\alpha_{2}} \eta, t^{-\alpha_{3}} \zeta\right)$ к уравнению (5.3). Из требования однородности получаем $\left\{\alpha_{1}, \alpha_{2}, \alpha_{3}\right\}=\{1,2,3\}$, т. е. начальный отрезок последовательности Вейерштрасса содержит тройку $\{1,2,3\}$ и, следовательно, $n>3$. 
В некоторых случаях условие $n>3$ является достаточным. По соображениям однородности полиномиальное соотношение веса 4 для ю-функций, если оно существует, всегда имеет вид

$$
\wp_{g, g, g, g}=6 \wp_{g, g}^{2}+a_{0} \wp_{g, g-2}+b_{0} \wp_{g-1, g-1}+a_{1} \wp_{g, g-1}+a_{2} \wp_{g, g}+a_{4},
$$

где индексы у параметров $a$ и $b$ равны их весам. $\mathrm{B}$ «рациональном пределе» $\boldsymbol{\lambda} \rightarrow \mathbf{0}$ параметры $a_{4}, a_{2}$ и $a_{1}$ принимают нулевые значения, а функция $\sigma(\boldsymbol{v}, \boldsymbol{\lambda})$ переходит в специальный полином Шура $\sigma_{n, s}(\boldsymbol{v})$ - полином ШураВейерштрасса (см. [4]). Как известно [9], удвоенная вторая производная логарифма любого полинома Шура по переменной веса 1 и, в частности, рациональный предел функции $2 \wp_{g, g}$, является рациональным решением уравнения (5.3), откуда следует $a_{0}=-4$ и $b_{0}=3$.

$\mathrm{K}$ сожалению, ограниченный объем статьи не позволяет нам расширить список примеров. Мы надеемся, что приведенные выше результаты достаточно ясно демонстрируют естественность нашего подхода, основанного на $(n, s)$ моделях кривых и теории ю-функций многих переменных, в контексте приложения теории абелевых функций к интегрированию нелинейных дифференциальных уравнений.

\section{Литература}

1. Baker H. F. Multiply Periodic Functions. Cambridge University Press, Cambridge, 1907.

2. Baker H. F. Abelian Functions. Cambridge University Press, Cambridge, 1995.

3. Buchstaber V. M., Enolskii V. Z., Leykin D. V. Kleinian functions, hyperelliptic Jacobians and applications. In: Reviews in Mathematics and Mathematical Physics (Novikov S. P., Krichever I. M., eds.), vol. 10, no. 2, Gordon \& Breach, London, 1997, pp. $1-125$.

4. Бухштабер В. М., Лейкин Д. В., Энольский В. З. Рациональные аналоги абелевых функций. Функц. анализ и его прил., 33, вып. 2, 1-15 (1999).

5. Buchstaber V. M., Rees E. G. Symmetric Powers of Algebraic Varieties. Preprint, Edinburgh University, Dec. 1999.

6. Дубровин Б. А. Тета-функции и нелинейные уравнения. УМН, 36, вып. 2, 11-80 (1981).

7. Fay J. D. Theta functions on Riemann surfaces. Lecture Notes in Math., vol. 352, Springer-Verlag, Berlin, 1973.

8. Гантмахер Ф. Р. Теория матриц. Наука, М., 1967.

9. Кач В. Г. Бесконечномерные алгебры Ли. Мир, М., 1993.

10. Klein F. Über hyperelliptische Sigmafunctionen. Math. Ann., 32, 351-380 (1888).

11. Мамфорд Д. Лекции о тета-функциях. Мир, М., 1988.

12. Чеботарев Н. Г. Теория алгебраических функций. ОГИЗ, М., 1948.

Московский государственный университет

им. М. В. Ломоносова

Поступило в редакцию

e-mail: buchstab@mech.math.msu.su

22 мая 2000 г.

Институт магнетизма НАНУ, Киев

e-mail: dile@imag.kiev.ua

Институт магнетизма НАНУ, Киев

e-mail: vze@imag.kiev.ua 